\title{
Open reduction of zygoma fractures with the extended transconjunctival approach and T-bar screw reduction
}

\author{
Seung Han Song ${ }^{1}$, Hyeokjae Kwon ${ }^{1}$, Sang-Ha Oh${ }^{1}$, Sun-Je Kim², Jaebeom Park ${ }^{3}$, Su Il Kim ${ }^{4}$ \\ ${ }^{1}$ Department of Plastic and Reconstructive Surgery, Chungnam National University College of Medicine, Daejeon; ${ }^{2}$ Muju Public Health and \\ Medical Care Center, Muju; ${ }^{3}$ Imsil Public Health and Medical Care Center, Imsil; ${ }^{4}$ Department of Anatomy, Chungnam National University \\ College of Medicine, Daejeon, Korea
}

Background Zygomaticomaxillary complex (ZMC) fractures mostly occur in the form of tripod fractures. The surgical field is accessed using a combination of three classic approaches. However, the subciliary incision may have unfavorable aesthetic results. Herein, the authors report the advantages of the extended transconjunctival approach (ETA) combined with T-bar screw reduction in minimizing scarring and complications for the treatment of ZMC fractures. Methods A total of 26 patients underwent ZMC reduction through the ETA and intraoral approach. A skin incision measuring roughly 5 to $8 \mathrm{~mm}$ in length was placed following the lateral canthal skin crease. After releasing the inferior crus of the lateral canthal tendon for canthotomy, the medial periosteum of the lateral orbital rim was preserved for canthal reattachment. A limited subperiosteal dissection and partial relaxing incision of the orbicularis oculi were performed to expose the fracture line of the inferior orbital rim and zygomaticofrontal suture. Reduction was performed using a T-bar screw through the transconjunctival incision and an elevator through the intraoral incision.

Results The aesthetic and functional results were excellent. Successful reduction was achieved and the skin incision was less than $8 \mathrm{~mm}$ in 20 cases (76.9\%). Only six patients had an additional skin incision (less than $5 \mathrm{~mm}$ ) to achieve reduction. No cases of ectropion, entropion, or excessive scarring were noted.

Conclusions The ETA using a T-bar screw is a useful method for maximizing aesthetic results in ZMC fractures, with the advantages of minimal scarring, faster recovery, and maintenance of pretarsal fullness.

Keywords Zygomatic fractures / Zygoma / Facial bones / Cicatrix
Correspondence: Seung Han Song Department of Plastic and Reconstructive Surgery, Chungnam National University College of Medicine, 282 Munhwa-ro, Jung-gu, Daejeon 35015, Korea

Tel: $+82-42-280-7380$

Fax: +82-42-280-7384

E-mail: silverwine_@naver.com

Received: 12 Apr $2018 \bullet$ Revised: 3 Jun $2018 \bullet$ Accepted: 18 Jun 2018

pISSN: 2234-6163 • elSSN: 2234-6171 • https://doi.org/10.5999/aps.2018.00311 • Arch Plast Surg 2018;45:325-332

\section{INTRODUCTION}

In most patients with a zygomatic complex fracture, the zygomaticofrontal region is treated through a lateral brow incision, and the orbital floor and rim are usually approached by a subciliary or infrapalpebral incision.

The main disadvantages of these approaches are a visible scar and a relatively high incidence of postoperative lid malposition, 
such as ectropion. In particular, subciliary incision injures the orbicularis oculi muscle of the lower lid and may cause unfavorable aesthetic results by reducing pretarsal fullness.

In the ophthalmologic area, the extended transconjunctival approach (ETA) with a lateral canthotomy or paracanthal incision has widely been used for the reduction of orbital floor fractures. This approach offers exposure of the orbital floor and inferior orbital rim, while avoiding the risk of scleral show and ectropion. However, the most commonly presented criticism of this technique is limited exposure.

The zygomaticofrontal suture is often located at a lower level than many surgeons expect, and can be accessed by a lateral canthal incision. A few studies have investigated zygoma reduction using the ETA, but details of the surgical technique and longterm results have not been well described $[1,2]$ and some authors made a lateral canthal skin incision exceeding 1.0 to 2.0 $\mathrm{cm}[2,3]$. Furthermore, in some studies, invasive procedures, such as drill-hole canthoplasty or extensive periosteal dissection, were used $[4,5]$.

The purpose of this article is to report the ETA using a T-bar screw as a way to minimize scar formation in the treatment of zygoma fractures. Additionally, we describe technical details for reducing the incidence of conspicuous scarring and postoperative complications.

\section{METHODS}

A cadaver study was performed to determine the efficacy of ETA using a T-bar screw with limited incision and dissection in the treatment of zygomaticomaxillary complex fractures. Two fresh cadaver faces were dissected and studied before the clinical application of the techniques presented herein on patients. Between May 2012 and March 2016, 26 patients with a non-comminuted zygomatic complex fracture were treated using this technique. All patients had either an isolated zygoma tripod fracture or one associated with other facial fractures, and all were mainly treated by ETA with an intraoral incision and 3-point fixation. A T-bar screw was also used in all cases. The decision to proceed with open reduction and miniplate fixation was based on computed tomographic and clinical findings. Patients with severely comminuted fractures around the zygomaticofrontal suture or with extensive trauma of the midface (i.e., LeFort II or III fractures) were excluded.

Follow-up three-dimensional (3D) computed tomography was performed to evaluate the degree of reduction. All clinical photographs were taken with a digital camera (610D, Nikon, Tokyo, Japan) in a standardized manner. Scarring, ectropion, entropion, scleral show, and zygoma symmetry were evaluated.
Preoperative and postoperative photographs were given to two plastic surgeons for scar grading; where $0=$ no scar, $1=a$ minimal scar, $2=$ a moderate scar, and $3=$ an obvious scar. To prevent bias, the two plastic surgeons were separate individuals who did not participate in these operations. Patients performed a subjective self-evaluation using the same grading system. Functional outcomes were also evaluated by these two plastic surgeons.

\section{Surgical techniques}

Under general anesthesia, a gingivobuccal incision was made 1 $\mathrm{cm}$ above the attached gingiva, and the buccinators and periosteum were incised and reflected superiorly as a mucoperiosteal flap. The zygomaticomaxillary buttress and posterior surface of the zygoma were exposed by intraoral incision. A lateral canthal extension mark, measuring approximately 5 to $8 \mathrm{~mm}$, was drawn on the lateral aspect of the lateral canthal angle following a skin crease. In the lateral canthal region, skin and the orbicularis oculi muscle were incised horizontally and a lateral canthal incision was made down the periosteum of the lateral orbital rim (Fig. 1). A lateral canthotomy was performed and the inferior crus of the lateral canthal tendon was released. After this procedure, the lower eyelid was everted and the incision was extended through the conjunctiva. The transconjunctival incision was made at $3 \mathrm{~mm}$ below the tarsus, extending from the lateral commissure to a point 3 to $4 \mathrm{~mm}$ lateral to the lacrimal punctum. The dissection proceeded anteriorly and inferiorly between the orbicularis oculi muscle and orbital septum (preseptal plane) until the periosteum of the inferior orbital rim was exposed. The lateral aspect of the upper lid was retracted superiorly through the lateral canthal incision, and the periosteum of the lateral orbital rim was exposed. The periosteum was incised and the orbital floor, the inferior orbital rim, and the lateral orbital rim, including the zygomaticofrontal suture area, were exposed by limited subperiosteal dissection. The periosteum of the lateral orbital rim was then elevated laterally to expose the fracture line and zygomaticofrontal suture. The medial periosteum of the lateral orbital rim was preserved for canthal reattachment when closing (Fig. 2).

If the exposure was not enough to reach the zygomaticofrontal suture, the orbicularis oculi muscle above the lateral canthal incision was incised upward vertically to provide sufficient exposure of the fractured zygomaticofrontal suture (Fig. 3).

During the procedures, only a small width of periosteum around the fracture line was dissected for screw fixation, and the periosteum of the other, non-fractured portion was preserved as much as possible (Fig. 4). A T-bar screw was used for repositioning the fractured zygoma in all cases. The malar eminence 


\section{Fig. 1. Cadaver study and diagram of the ETA}

(A) Cadaver study demonstrating the extended transconjunctival approach (ETA). Note that the zygomaticofrontal suture (red dotted line) and the lateral orbital rim are sufficiently exposed for miniplate fixation. (B) Diagram of periosteal dissection around the lateral orbital rim. The medial periosteum on the lateral orbital rim is preserved.
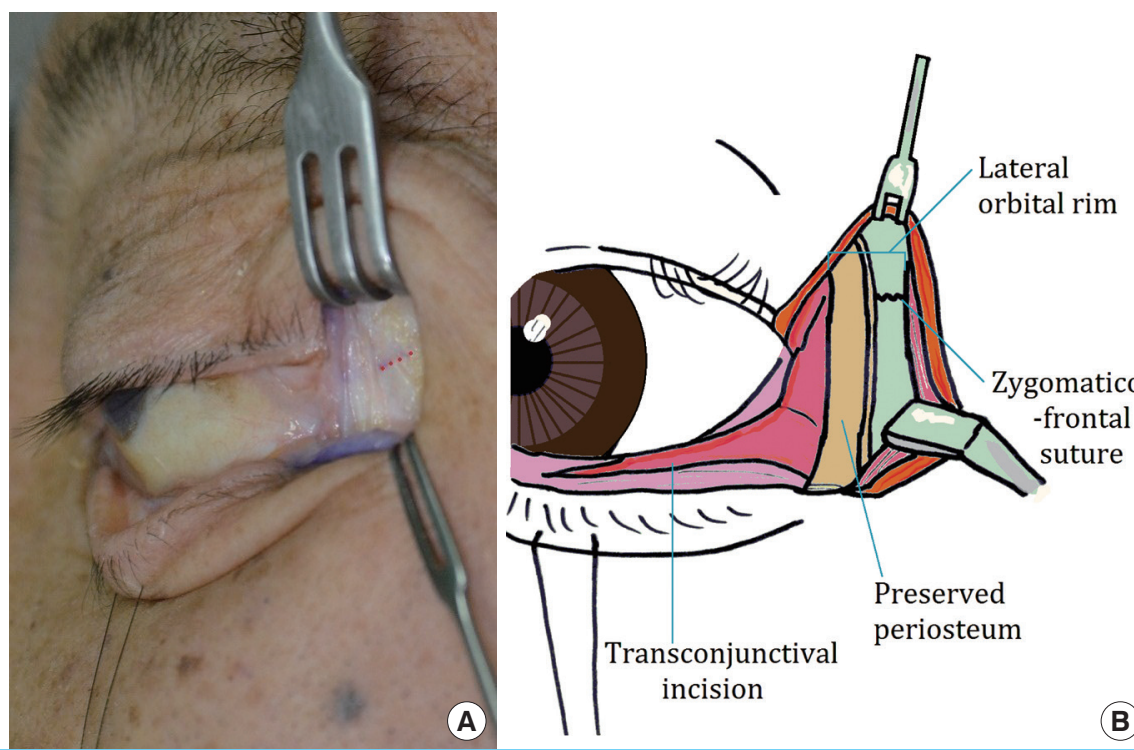

\section{Fig. 2. Cadaver dissection and diagram of lateral rim}

(A) For canthal reattachment, the medial portion of the anterior aspect of the periosteum was preserved. This allows invasive procedures, such as drill-hole canthoplasty, to be avoided. (B) Diagram of the suture position of the periosteum on the lateral orbital rim.

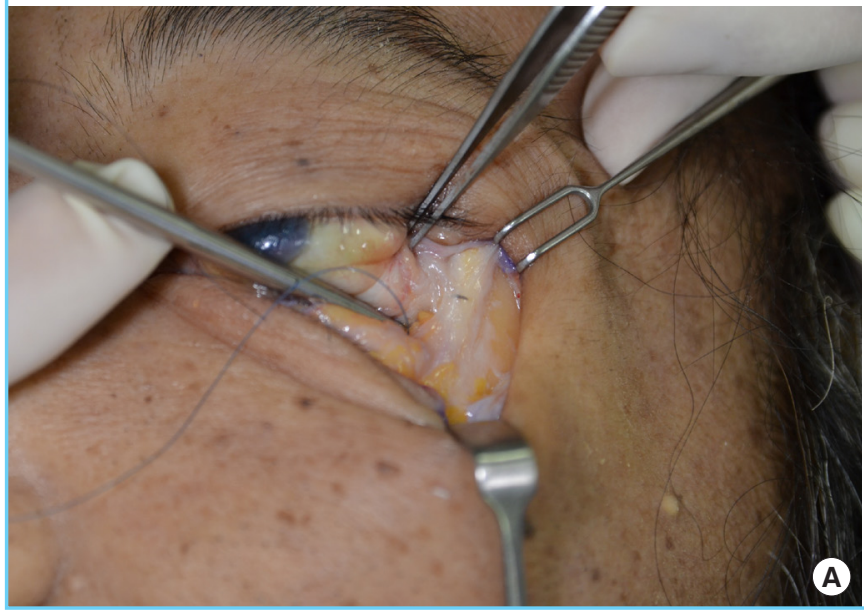

was penetrated using a perforating drill, a 2.4-mm bit, and a drill guide. The T-bar screw was introduced into the hole produced and rotated in a clockwise direction until the screw was firmly fixed within the bone (Fig. 5). At this point, a Dingman elevator was placed intraorally underneath the arch. The handle on the T-bar screw was then used to grasp and control the screw as necessary to provide counter-vectors of the embedded zygomatic bone in three dimensions. With direct visualization of the fractured site through a transconjunctival incision, we reduced the zygomatic bone into its anatomic position by pulling the T-bar handle in the required direction. Reduction with the Dingman elevator through an intraoral incision was also combined with T-bar manipulation.

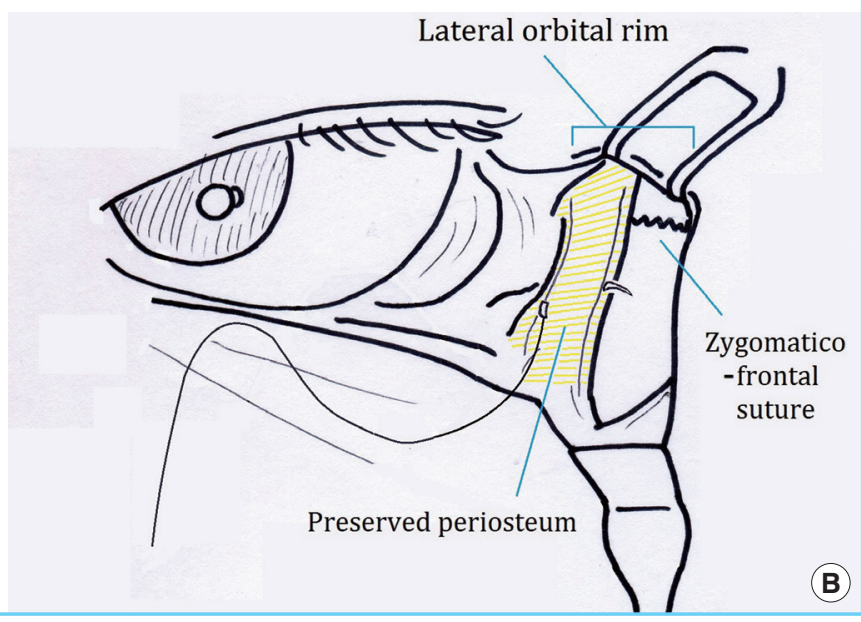

If the zygoma reduction was not sufficient using the above techniques, the skin incision was extended laterally by about 5 $\mathrm{mm}$, and a bone elevator was placed posteriorly to the body of the zygoma along the zygomatic arch, through the extended lateral canthal skin incision, and reduction was then performed. An additional extension of the lateral canthal skin incision was needed in only six patients (23.1\%).

In all cases, complete reduction was confirmed by accurate alignment of the lateral and inferior orbital walls. Then, the 3 points (zygomaticofrontal suture, inferior orbital rim, and the zygomaticomaxillary buttress) were fixated with titanium miniplates and screws. If there was an associated blow-out fracture, orbital floor reconstruction with a porous polyethylene implant 


\section{Fig. 3. Relaxing incision of orbicularis oculli muscle}

(A) Diagram demonstrating exposure of the zygomaticofrontal suture. (B) A partial relaxing incision of the orbicularis oculi muscle is made upward vertically. (C) By this technique, adequate exposure for miniplate fixation can be obtained.

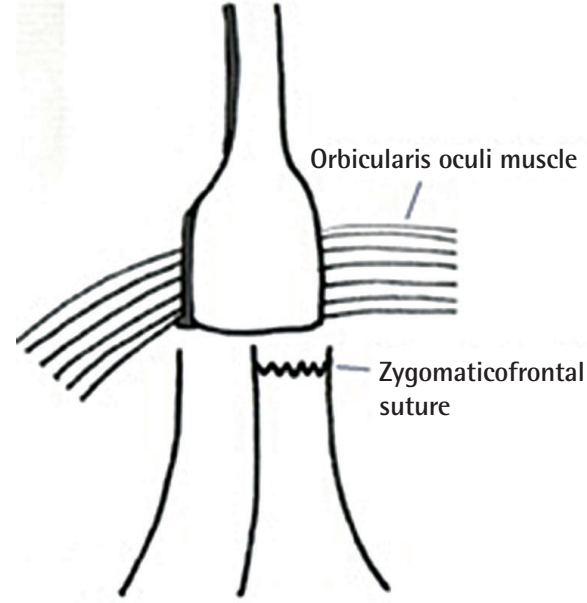

Lateral orbital rim
(A)

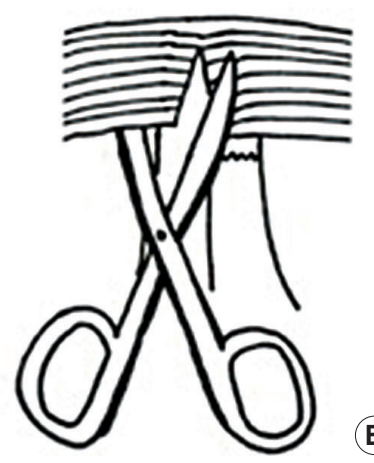

(B)

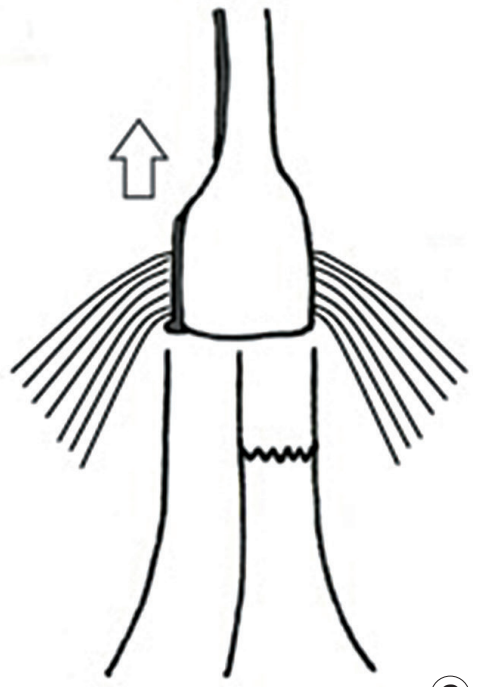

(C)

\section{Fig. 4. Cadaver dissection of the inferior orbital rim}

Only a small width of periosteum around the fracture line was dissected. To minimize invasiveness, the periosteum of the non-fractured inferolateral portion (yellow arrow) was preserved as much as possible.

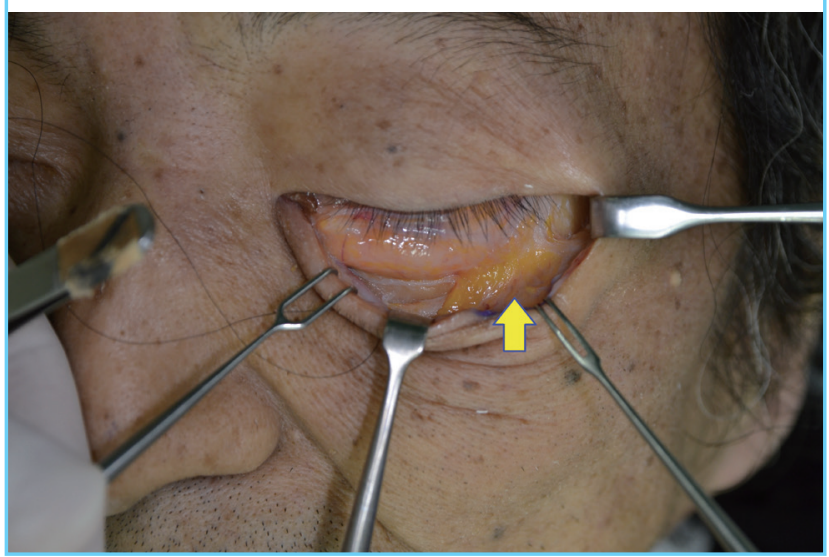

(Synpor) was performed. The periosteum was closed with a 5-0 absorbable suture, and canthal reattachment was accomplished by 5-0 absorbable suture fixation in the posterior-superior direction to the preserved periosteum of the lateral orbital rim to prevent postoperative ectropion.

The lateral portion of the lower lid orbicularis oculi muscle flap was suspended on the periosteum of the lateral orbital rim for additional canthal support. Finally, the skin and subcutaneous layer at the lateral canthal incision site were closed with a 6-0 suture. The intraoral incision site was closed with a 4-0 absorbable suture. A temporary tarsorrhaphy suture (antichemosis

\begin{tabular}{|lc|}
\hline \multicolumn{2}{|c|}{ Table 1. Patient data } \\
\hline \multicolumn{1}{|c|}{ Characteristics } & Value \\
\hline No. of patients & 26 \\
$\quad$ Male & 21 \\
Female & 5 \\
Age, mean (range), yr & $31(17-58)$ \\
Cause & \\
$\quad$ Fights & 12 \\
Traffic accident & 5 \\
Sports-related injury & 4 \\
Falling down & 3 \\
Industrial accident & 2 \\
\hline
\end{tabular}

suture) was placed to prevent postoperative chemosis.

\section{RESULTS}

Of the 26 patients, 21 were men and five were women. The patients' mean age was 31 years (range, $17-58$ years). Twenty-one $(80.7 \%)$ had an associated orbital floor fracture and three (11.5\%) had an associated nasal bone fracture. The injury mechanisms included three cases of falling down, five traffic accidents, 12 fights, four sports-related injuries, and two industrial accidents (Table 1). Patients were followed up for 3 to 37 months, and the mean follow-up was 23.5 months.

Twenty patients (76.9\%) were treated using only the ETA technique and the intraoral approach. The skin incision did not exceed 5 to $8 \mathrm{~mm}$ in any of those 20 cases. Reduction with a Dingman elevator through an additional extension of the lateral 
canthal skin incision $(3-5 \mathrm{~mm})$ was performed in only six patients (23.1\%). All patients had aesthetically satisfactory results. There were no cases of facial asymmetry, lateral canthal displacement, entropion, ectropion, diplopia, enophthalmos, or extraocular muscle dysfunction, and no patient showed functional impairment. Two patients (7.7\%) complained of persistent chemosis, but this resolved after 3 months of conservative care. Three patients (11.5\%) had mild hyperpigmentation at the lateral canthal incision site postoperatively. However, the scarring was improved by several sessions of laser therapy. Most patients $(88.5 \%)$ showed no visible scar in the skin incision area (Fig. 5).

Zygomatic symmetry was checked by postoperative radiography (X-ray, 3D facial computed tomography) and confirmed in all patients. In addition, computed tomography revealed satisfactory reduction of the zygomaticofrontal buttress, zygomaticomaxillary buttress, zygomatic arch, and inferior orbital rim in all patients. No significant asymmetry was noted in this series (Fig. 6).

The average scar grade as determined by the two plastic surgeons was 0.79 (evaluator 1, 0.88; evaluator 2, 0.69) and the average patient self-assessed scar grade was 0.50 . The patients appeared to be more satisfied with the results of surgery than the evaluators, but the differences between these groups were not statistically significant (Table 2).

\section{DISCUSSION}

The treatment of zygomatic complex fractures is frequently a challenge for plastic surgeons. The zygoma and maxilla provide contour to the face, and their symmetry is cosmetically important. Many surgical approaches have been introduced for the reduction of zygomatic complex fractures, including the intraoral (Keen), temporal (Gillies), brow incision, and bicoronal ap-

\section{Fig. 5. Intraoperative photograph and scar after surgery}

(A) We used a T-bar screw through an extended transconjunctival incision. The length of the lateral skin incision varied according to surgical needs, but was mostly within $8 \mathrm{~mm}$. (B) The scar was hardly visible at 3 months after surgery.
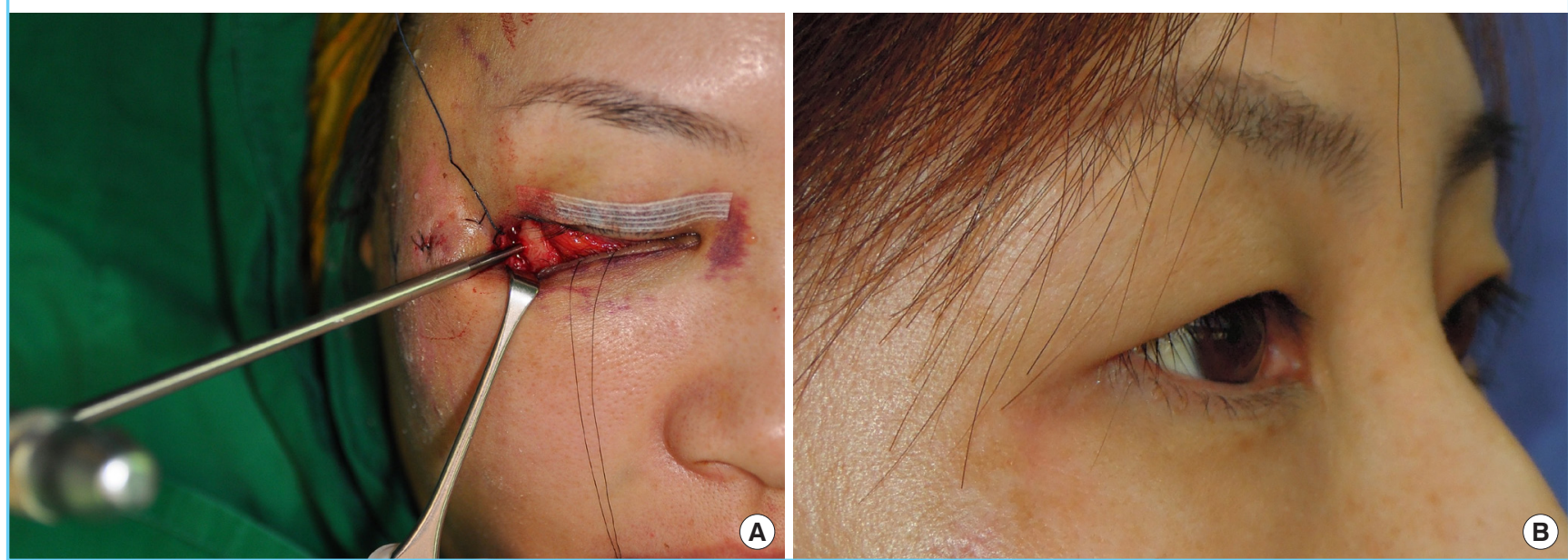

\section{Fig. 6. Preoperative and postoperative 3D-reconstructed CT images}

(A) Preoperative three-dimensional (3D) reconstructed computed tomographic (CT) images showing a zygoma tripod fracture. (B) Postoperative image showing anatomic reduction and fixation of the zygomaticomaxillary complex via the extended transconjunctival approach and the intraoral approach.
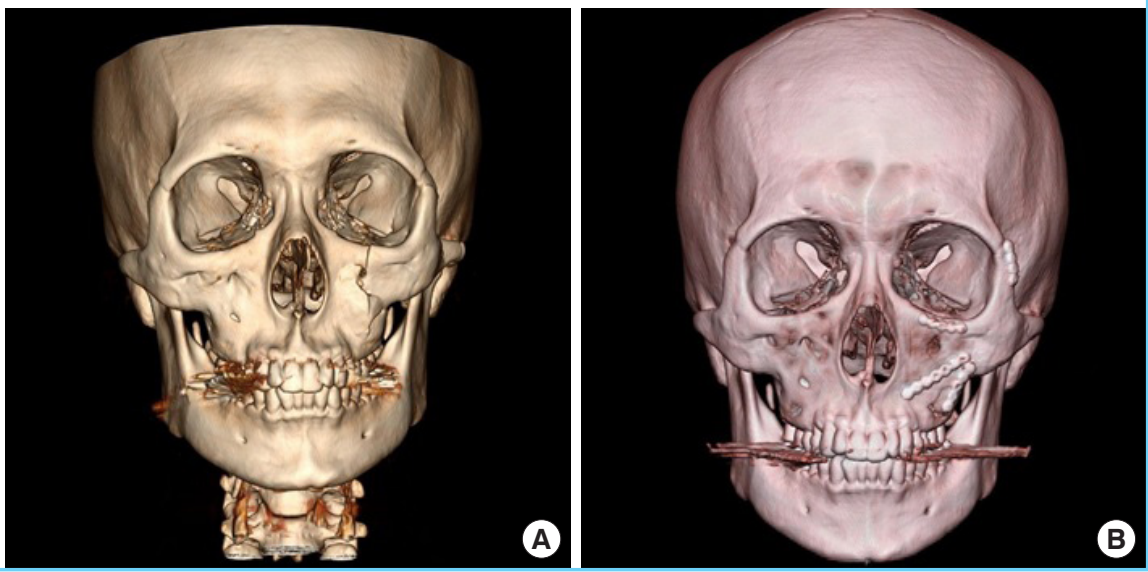


\begin{tabular}{|c|c|c|c|}
\hline & Evaluator 1 & Evaluator 2 & Patient \\
\hline \multicolumn{4}{|l|}{ Score } \\
\hline 0 & 6 & 11 & 15 \\
\hline 1 & 17 & 12 & 9 \\
\hline 2 & 3 & 3 & 2 \\
\hline 3 & 0 & 0 & 0 \\
\hline Average score & 0.88 & 0.69 & 0.5 \\
\hline
\end{tabular}

proaches $[1,2]$. The generally preferred approach consists of three incisions, including lateral brow and intraoral incisions. Although the subciliary incision, lateral brow incision, bicoronal approaches provide good exposure and direct visualization, they leave conspicuous external scars on the face. The temporal approach leaves no facial scars, but it cannot provide direct visualization, which may result in incomplete surgical reduction [2].

The intraoral approach also has the advantages of leaving no facial scars and being simple to use. Limited reductions are popular today; for example, the intraoral approach alone can be used for about $50 \%$ to $75 \%$ of all zygomatic complex fractures [6]. This type of limited approach can be used for fractures that are greensticked or minimal at the zygomaticofrontal suture, or include a minimal or linear orbital floor fracture that can be repaired by zygoma reduction. In most cases, however, one cannot fully assess the degree of reduction based on the intraoral approach alone, and further exposure is indicated [7].

The orbital floor and rim are usually approached by a subciliary or infrapalpebral incision $[2,8]$. However, these incisions leave a visible scar and have a risk of lower eyelid deformity. In particular, a subciliary incision can injure the orbicularis oculi muscle of the lower lid and decrease pretarsal fullness or cause aesthetically unfavorable results, such as lower eyelid retraction, both transient and permanent $[9,10]$.

The ETA is usually used in ophthalmology, and little information is available about treating zygoma complex fractures using the ETA approach; as such, its technical details and limitations are not well defined [1]. Lee et al. [2] reported 53 cases treated using the transconjunctival approach with a lateral canthal incision and 2-point fixations in all cases.

The present study differs in some respects from previous studies. In all 26 of our cases, an intraoral gingivobuccal incision was made to facilitate accurate reduction. While some previous reports have described 2-point fixation using a transconjunctival incision for the treatment of zygomatic fractures, in those studies, an intraoral approach was performed in few or no cases at all $[1,2]$. We have found that our method gives the surgeon an excellent view of 4 points of alignment (zygomaticofrontal suture, sphenozygomatic suture, inferior orbital rim, and the zygomaticomaxillary buttress). Our approach includes plating the zygomaticomaxillary buttress, which has been cited as an important component when planning reconstruction $[11,12]$.

For exposing the zygomaticofrontal suture, we recommend a lateral canthal incision of approximately 5 to $8 \mathrm{~mm}$ in most cases (76.9\%). During the ETA, the zygomaticofrontal suture can be accessed by a lateral canthal skin incision. The zygomaticofrontal suture is often located at a lower level than many surgeons expect. In cases of difficult exposure, a vertical incision on the orbicularis oculi muscle above the lateral canthus level was helpful for achieving sufficient exposure for fixation of the fractured zygomaticofrontal suture (Fig. 3). Because exposure of the zygomaticofrontal suture with a transconjunctival incision is more difficult than with a separate lateral brow incision, by using this technique, it is possible to avoid strong traction, which can result in more postoperative swelling [13].

We then used a T-bar screw through the extended transconjunctival incision, because reduction using a bone elevator through the lateral canthal incision has the disadvantage of involving a slightly inadequate vector for reduction of the zygoma. A fractured zygoma is usually displaced posteriorly and medially, so it should be manipulated by pulling it in the opposite direction, anteriorly and laterally [1]. However, the lateral canthal incision is located more medially than the lateral brow incision. The lateral brow incision can provide rotary force in the vertical axis, allowing reduction of a depressed zygoma, which is more difficult to reduce through the lateral canthal incision. Thus, we used a T-bar screw and intraoral approach to overcome this problem. The T-bar screw is easy to use and allows the precise 3D manipulation of zygomaticomaxillary complex fractures, because it is controllable in any direction. Reduction using a towel clip or bone elevator through the temporal or intraoral approach limits the ability of the surgeon to provide force or rotation along all directions in three dimensions $[14,15]$. Despite its advantages, many surgeons prefer not using a T-bar screw because they are accustomed to the conventional method, in which additional brow incisions are used to expose the zygomatic bone $[1,16]$. However, using a T-bar screw provides direct and concentrated force, enables depressed zygomatic fractures to be more precisely corrected, and allows the surgeon to avoid the need for a brow incision and the risk of excessive scars on the face.

If reduction with a T-bar screw and the intraoral approach is unsatisfactory, excessive attempts to insert the elevator through a small lateral canthal incision are inappropriate, because they leave crushing injuries and abrade skin around the lateral canthus. To overcome this problem, an additional extension of the lateral canthal skin incision by roughly $5 \mathrm{~mm}$ should be consid- 
ered. To place the bone elevator easily in the posterior aspect of the zygoma, the lateral canthal incision was extended by approximately $5 \mathrm{~mm}$ in six of our 26 cases (23.1\%). For canthal reattachment, periosteum over the medial portion of the lateral orbital rim is routinely preserved, which facilitates the adhesion of the lateral canthal tendon to the lateral orbital rim (Fig. 1). Using this method, invasive techniques such as drill-hole canthoplasty can be avoided, and non-fractured sites of the inferior orbital rim can be preserved to minimize the invasiveness of the approach and the recovery period after surgery. In cases of severely comminuted lateral orbital wall fractures, which were excluded from the present study, the medial periosteum of the lateral orbital rim cannot be preserved. In such cases, drill-hole canthoplasty can be considered to fix the lateral canthal tendon and to prevent postoperative ectropion. Before adopting the ETA technique, preoperative computed tomographic images should be carefully reviewed.

In this study, no conspicuous scarring was observed in most cases. Only three patients (11.5\%) had mild hyperpigmentation at the lateral canthal incision site in the early postoperative period. In all three of these patients, reduction was performed using a bone elevator through the lateral canthal incision, which was extended by approximately $5 \mathrm{~mm}$ along the initial skin incision. Reduction by a bone elevator through the lateral canthal incision may leave abrasions around the incision site. To prevent this problem, we placed gauze under the elevator during reduction. Additionally, before skin repair, the authors performed marginal skin debridement on the lateral canthal region.

Chemosis (conjunctival edema) occurs frequently after periorbital surgery, but in most cases, it resolves spontaneously [17]. However, a temporary tarsorrhaphy suture should be consid-

\section{Fig. 7. Technique to fix the tarsal plate}

Precise technique is required to place the needle of 4-0 Prolene on the tarsal plate as much as possible.

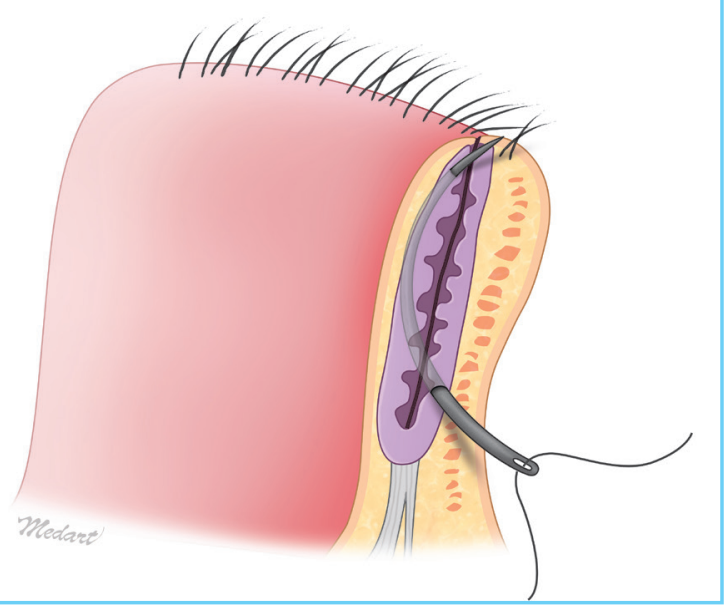

ered when significant dissection has been performed. Tarsorrhaphy can be used as a preventative measure to treat chemosis at any stage [18]. In the present study, two patients (7.7\%) complained of persistent chemosis, and in both patients, chemosis resolved after 3 months of conservative treatments, which included lubrication, ocular decongestants, and steroid drops.

The limitations of our method are. First, it is difficult to apply to comminuted fractures. If intact bone for T-bar screw fixation is not available in patients with comminuted fractures, this method may not be helpful for reduction. Although these fractures can be exposed through the ETA and an oral incision, multidirectional approaches that include a lateral brow incision may be required for complete reduction $[1,7]$. Second, a potential risk of the technique is lid malposition, such as ectropion, entropion, or canthal deformity. To prevent such complications, we performed the following techniques. We preserved the medial portion of periosteum on the lateral orbital rim for lateral canthal reattachment, which also facilitated recovery and lateral canthal tendon adhesion to the lateral orbital rim. For secure canthoplasty, it is important to perform accurate canthal anchoring by precisely tethering the lower lid tarsal plate to the medial portion of the lateral orbital rim. The lower lid tarsal plate is in the posterior layer of the lower lid, and its height is approximately 4 to $5 \mathrm{~mm}$ and its thickness is roughly $1.0 \mathrm{~mm}$. A precise technique is required to place the needle of 4-0 Prolene on the tarsal plate as much as possible (Fig. 7). We performed orbicularis oculi muscle suspension for reinforcement of canthal support (Fig. 8) [19].

Surgeons should carefully consider the surgical procedure and pay attention to the risk of lid retraction, and must know how to tighten a lateral canthal tendon and how to surgically correct

\section{Fig. 8. Orbicularis oculi suspension}

In all cases, orbicularis oculi suspension was performed before skin closure. The technique consists of suspending the muscle flap of the lower lid on the periosteum in the lateral orbit rim, and contributes to the prevention of lower lid malposition, such as ectropion.

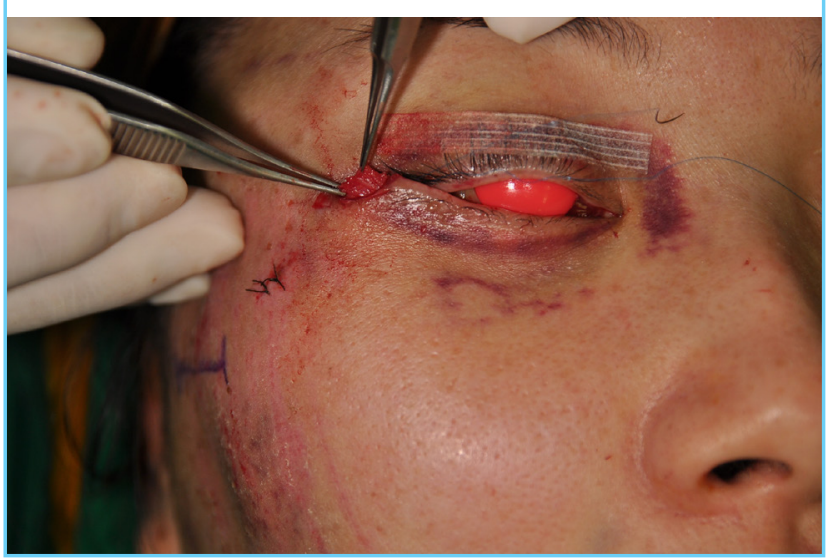


lower eyelid retraction.

After a mean follow-up of 23.5 months, we are confident that the proposed technique provides an excellent alternative for patients who are concerned about postoperative scarring. The lateral canthal incision was designed by following the skin crease, and it is much less noticeable than a lateral brow incision. In addition to inconspicuous scarring, it provides maintenance of pretarsal fullness and has few complications, such as lid malposition. In conclusion, we believe that the ETA using a T-bar screw provides excellent aesthetic results, such as minimal scarring and pretarsal fullness, in treating non-comminuted zygomaticomaxillary complex fractures.

\section{NOTES}

\section{Conflict of interest}

No potential conflict of interest relevant to this article was reported.

\section{Ethical approval}

The study was performed in accordance with the principles of the Declaration of Helsinki. Written informed consents were obtained.

\section{Patient consent}

The patients provided written informed consent for the publication and the use of their images.

\section{REFERENCES}

1. Chang EL, Hatton MP, Bernardino CR, et al. Simplified repair of zygomatic fractures through a transconjunctival approach. Ophthalmology 2005;112:1302-9.

2. Lee PK, Lee JH, Choi YS, et al. Single transconjunctival incision and two-point fixation for the treatment of noncomminuted zygomatic complex fracture. J Korean Med Sci 2006;21:1080-5.

3. Kim DW, Choi SR, Park SH, et al. Versatile use of extended transconjunctival approach for orbital reconstruction. Ann Plast Surg 2009;62:374-80.

4. Uemura T, Jinnai T, Yokoyama T, et al. C-shape extended transconjunctival approach for the exposure and osteotomy of traumatic orbitozygomaticomaxillary deformities. J Craniofac Surg 2001;12:603-7.
5. Martinez AY, Bradrick JP. Y modification of the transconjunctival approach for management of zygomaticomaxillary complex fractures: a technical note. J Oral Maxillofac Surg 2012;70:97-101.

6. Rodriguez ED, Dorafshar AH, Manson PN. Facial fraactures. In: Neligan PC, editor. Plastic surgery. 3rd ed. Philadelphia: Saunders Elsevier; 2013. vol. 3, p. 49-88.

7. Kaufman Y, Stal D, Cole P, et al. Orbitozygomatic fracture management. Plast Reconstr Surg 2008; 121:1370-4.

8. Manganello-Souza LC, Rodrigues de Freitas R. Transconjunctival approach to zygomatic and orbital floor fractures. Int J Oral Maxillofac Surg 1997;26:31-4.

9. Holtmann B, Wray RC, Little AG. A randomized comparison of four incisions for orbital fractures. Plast Reconstr Surg 1981;67:731-7.

10. Werther JR. Cutaneous approaches to the lower lid and orbit. J Oral Maxillofac Surg 1998;56:60-5.

11. Yamamoto Y, Kawashima K, Sugihara T, et al. Surgical management of maxillectomy defects based on the concept of buttress reconstruction. Head Neck 2004;26:247-56.

12. Tarabichi M. Transsinus reduction and one-point fixation of malar fractures. Arch Otolaryngol Head Neck Surg 1994; 120:620-5.

13. Manson PN, Ruas E, Iliff N, et al. Single eyelid incision for exposure of the zygomatic bone and orbital reconstruction. Plast Reconstr Surg 1987;79:120-6.

14. Kreutziger KL, Kreutziger KL. Zygomatic fractures: reduction with the T-bar screw. South Med J 1992;85:1193-202.

15. Karlan MS, Cassisi NJ. Fractures of the zygoma: a geometric, biomechanical, and surgical analysis. Arch Otolaryngol 1979;105:320-7.

16. BaekJE, Chung CM, Hong IP. Reduction of zygomatic fractures using the carroll-girard T-bar screw. Arch Plast Surg 2012;39:556-60.

17. Woo KI, Choi CY. High-frequency radiowave electrosurgery for persistent conjunctival chemosis following cosmetic blepharoplasty. Plast Reconstr Surg 2014;133:1336-42.

18. Weinfeld AB, Burke R, Codner MA. The comprehensive management of chemosis following cosmetic lower blepharoplasty. Plast Reconstr Surg 2008;122:579-86.

19. Hester TR Jr. Evolution of lower lid support following lower $\mathrm{lid} /$ midface rejuvenation: the pretarsal orbicularis lateral canthopexy. Clin Plast Surg 2001;28:639-52. 